\title{
Determination of Zearalenone in Urine Samples from Guatemala: A Pilot Assessment
}

\author{
Christian S. Alvarez*1, Sean Burke*2, Patricia Zaid ${ }^{3}$, Joshua W. Smith², Alvaro \\ Rivera-Andrade $^{4}$, María F. Kroker-Lobos ${ }^{4}$, Manuel Ramírez-Zea ${ }^{4}$, Michael Dean ${ }^{1}$, \\ Katherine A. McGlynn ${ }^{1}$, and John D. Groopman ${ }^{2,5}$ \\ ${ }^{1}$ Division of Cancer Epidemiology and Genetics, National Cancer Institute, Rockville, MD, \\ U.S.A. \\ ${ }^{2}$ Department of Environmental Health and Engineering, Bloomberg School of Public \\ Health, Johns Hopkins University, Baltimore, MD, U.S.A. \\ ${ }^{3}$ Tecnicas Biológicas, S.A. Biotec, Guatemala City, Guatemala \\ ${ }^{4}$ INCAP Research Center for the Prevention of Chronic Diseases, Institute of Nutrition of \\ Central America and Panama, Guatemala City, Guatemala \\ ${ }^{5}$ Department of Epidemiology, Bloomberg School of Public Health, Johns Hopkins \\ University, Baltimore, MD, U.S.A.
}

February 11, 2022

*Co-first authors

\begin{abstract}
:
Zearalenone (ZEA), one of the most prevalent estrogenic mycotoxins, contaminates crops, including maize, rice, and wheat. Previous studies have found high levels of mycotoxins, particularly fumonisin $\mathrm{B}_{1}\left(\mathrm{FB}_{1}\right)$ and aflatoxin $\mathrm{B}_{1}\left(\mathrm{AFB}_{1}\right)$ in maize from Guatemala. In addition, our group has reported high levels of serum aflatoxin $\mathrm{B} 1\left(\mathrm{AFB}_{1}\right)$ in this population. However, exposure to ZEA has not been examined in Guatemala. Thus, this pilot study assessed the presence of ZEA in urine samples from Guatemala City and vicinity $(\mathrm{n}=50)$. In addition, correlation between $\mathrm{ZEA}$ and $\mathrm{AFB}_{1}$ was examined as well as correlations with maize and tortilla consumption. Ten of the 50 samples had detectable levels of ZEA, with a median level of 2.0 $\mathrm{pg} / \mathrm{uL}$. No correlations were found with $\mathrm{AFB}_{1}$ levels, nor with maize or tortilla consumption. This is the first report on the presence of ZEA in urine samples from Guatemala. These results suggest that further research on ZEA in Guatemala is warranted.
\end{abstract}

\section{Background:}

Zearalenone (ZEA), a nonsteroidal mycotoxin with estrogenic properties, is produced by several species of Fusarium fungi that contaminate crops, such as maize, rice, wheat, barley, sorghum, and oats. ${ }^{1}$ The estrogenic effects of ZEA cause a variety of reproductive disorders in animals, especially in swine. ${ }^{2}$ ZEA has also been reported to increase the risk of liver and pituitary cancers in mice. ${ }^{3}$

There is limited data examining exposure to ZEA using biological samples. In rural China, a study that examined the presence of several mycotoxins in urine and plasma samples $(n=260)$ found that ZEA was one of the most prevalent mycotoxins detected for both type of samples. ${ }^{4}$ In addition, a recent study from Spain $(n=40)$ reported that $72.5 \%$ of urine samples had at least one of the mycotoxins examined, with ZEA being present in $40 \%$ of the samples. ${ }^{5}$ Correlations between ZEA and other mycotoxins (e.g. aflatoxin) have 
not been examined in these studies, nor have correlations with dietary data been conducted to determine potential sources of contamination.

In Guatemala, a 2012 survey found high levels of mycotoxins, particularly fumonisin $\mathrm{B}_{1}\left(\mathrm{FB}_{1}\right)$ and aflatoxin $\mathrm{B}_{1}\left(\mathrm{AFB}_{1}\right)$ in maize samples across the country. ${ }^{6}$ In 2017 , our group reported high levels of serum $\mathrm{AFB}_{1^{-}}$ albumin adducts in a cross-sectional study of Guatemalan adults. ${ }^{7}$ To the best of our knowledge, ZEA has not been assessed in biological samples in this population. Therefore, the purpose of this pilot study was to determine the presence of ZEA in urine samples from individuals in Guatemala. In addition, the current study aimed to assess the correlation between urinary levels of ZEA and serum levels of $\mathrm{AFB}_{1}$-albumin adducts, and to assess the associations between ZEA and maize and tortilla consumption levels.

\section{Methods:}

In 2019, urine and blood samples were obtained from 25 men and 25 women aged 18 years and older from Guatemala City and vicinity. The sample size was selected to capture a sufficient range of possible detectable levels of ZEA and $\mathrm{AFB}_{1}$-lys albumin adducts. Demographic information such as age, sex, residence, as well as maize consumption was available. The determinations of $\mathrm{AFB}_{1}$-lys adducts and ZEA levels were performed by isotope-dilution mass spectrometry in the laboratory of Dr. John D. Groopman at the Johns Hopkins University Bloomberg School of Public Health. AFB 1 adduct concentrations were normalized to total serum albumin and expressed as pg $\mathrm{AFB}_{1}$-lys adduct/mg albumin. ${ }^{7}$

Descriptive statistics were used to characterize the individuals overall and stratified by sex. Spearman rank-order correlation coefficients were calculated to assess the correlation between urinary levels of ZEA and serum levels of $\mathrm{AFB}_{1}$-albumin adducts. In addition, correlations between ZEA and maize and tortilla consumption levels were also computed. A two-sided p-value $<0.05$ was considered statistically significant. The statistical analysis was conducted using SAS v.9.4 (SAS Institute, Cary, NC).

\section{Results:}

The median age of the individuals was 34 years (Interquartile range [IQR]: 26,43). Over 50\% of the samples were from persons who resided in Guatemala City and vicinity. The median maize consumption among men was 443.4 grams per day (IQR: 166.5, 755.2) and among women was 245.3 grams per day (IQR: 51.3, 559.6). In addition, the median tortilla consumption, the main foodstuff in the Guatemalan diet, among men was 375.0 grams per day (IQR: 150.8, 754.2) and among women was 71.5 grams per day (IQR: 51.3, 133.0) (Table 1 ).

Nearly all samples $(\mathrm{n}=49)$ had detectable $\mathrm{AFB}_{1}$-lys adduct levels, with a median of $4.3 \mathrm{pg} / \mathrm{mg}$ (IQR: 2.0, 9.0). Ten of the 50 samples had detectable levels of ZEA, with a median level of $2.0 \mathrm{pg} / \mathrm{uL}$ (Table $\mathbf{1}$ ). No significant correlation was observed between urinary levels of ZEA and serum levels of AFB $_{1}$-albumin adducts (Spearman's rank correlation coefficient test $=0.0014 ; \mathrm{p}$ value: 0.99$)$ (Figure 1 ). In addition, no significant correlations between ZEA levels and maize or tortilla consumption were found (data not shown).

\section{Conclusions:}

This is the first report on the presence of ZEA in urine samples from Guatemala. These results suggest that further research on ZEA in Guatemala is warranted.

\section{Conflict of interest:}

The authors declare no potential conflict of interest.

\section{References:}

1. Gupta RC. Veterinary Toxicology Basic and Clinical Principles Third Edition Preface. Veterinary Toxicology: Basic and Clinical Principles, 3rd Edition. 2018:Xxxi-Xxxii.

2. Pitt JI. Mycotoxins: Zearalenone. In: Encyclopeida of Food Safety. Vol 2. Elsevier; 2014:313-314.

3. Improving public health through mycotoxin control.International Agency for Research on Cancer; 2012. 
4. Fan K, Xu J, Jiang K, et al. Determination of multiple mycotoxins in paired plasma and urine samples to assess human exposure in Nanjing, China. Environ Pollut. 2019;248:865-873. doi:10.1016/j.envpol.2019.02.091.

5. Carballo D, Pallarés N, Ferrer E, Barba FJ, Berrada H. Assessment of human exposure to Deoxynivalenol, Ochratoxin A, Zearalenone and their metabolites biomarker in urine samples using LC-ESI-qTOF. Toxin (Basel). 2021;13(8):530.

6. Torres O, Matute J, Gelineau-van Waes J, et al. Human health implications from co-exposure to aflatoxins and fumonosins in maize-based foods in Latin America: Guatemala as a case study. World Mycotoxin Journal. 2015;8(2):143-159.

7. Smith JW, Kroker-Lobos MF, Lazo M, et al. Aflatoxin and viral hepatitis exposures in Guatemala: Molecular biomarkers reveal a unique profile of risk factors in a region of high liver cancer incidence.PLoS One. 2017;12(12):e0189255.

Table 1. Overall characteristics of the sample

\begin{tabular}{|c|c|c|c|}
\hline Characteristics & Total $(n=50)$ & Men $(n=25)$ & Women $(n=$ \\
\hline Age (median [IQR]) & $34(26,43)$ & $30(26,39)$ & $38(26,53)$ \\
\hline \multicolumn{4}{|l|}{ Residence, $\mathbf{n}(\%)$} \\
\hline Guatemala City and vicinity & $29(58 \%)$ & $11(44.0 \%)$ & $18(72.0 \%)$ \\
\hline Outside Guatemala City & $21(42 \%)$ & $14(56.0 \%)$ & $7(28.0 \%)$ \\
\hline Total maize consumption per day, grams, median (IQR) & $302.9(88.4,658.9)$ & $443.4(166.5,755.2)$ & $245.3(51.3$, \\
\hline Tortilla consumption per day, grams, median (IQR) & $134.4(66.7,437.5)$ & $375.0(150.8,754.2)$ & $71.5(51.3,1$ \\
\hline $\mathrm{AFB}_{1}$-albumin adduct levels, (median [IQR]) & $4.3(2.0,9.0)$ & $4.1(2.3,13.2)$ & $4.3(1.6,7.1)$ \\
\hline ZEA pg/uL (median $[\mathrm{IQR}])^{1}$ & $0.20(0.16,0.29)$ & $0.29(0.20,0.30)$ & $0.16(0.16,0$ \\
\hline Albumin mg/ml (median [IQR]) & $44.7(42.6,47.6)$ & $47.0(45.1,48.6)$ & $42.9(42.2,4$ \\
\hline Creatinine mg/dl (median [IQR]) & $109.1(68.9,146.3)$ & $115.6(86.4,210.0)$ & $95.1(68.9,1$ \\
\hline
\end{tabular}

${ }^{1} 10$ samples above the limit of quantification ( 5 among men and 5 among women) and most of the samples $(n=7)$ were from outside Guatemala City and vicinity.

Figure 1. Correlation between serum $\mathrm{AFB}_{1}$-albumin adduct levels and urinary

ZEA concentrations

\section{Hosted file}

Figure 1.jpg available at https://authorea.com/users/458783/articles/555253-determination-ofzearalenone-in-urine-samples-from-guatemala-a-pilot-assessment

Spearman's rank correlation coefficient test: 0.001; p value: 0.99 (No correlation).

Note: Removal of the outlier in the upper right corner of the figure did not result in better correlation (Spearman's rank correlation test $=-0.084$; $\mathrm{p}$ value 0.57 ). 